\title{
ADOPCIÓN DE ESTRATEGIAS EN PLATAFORMAS DIGITALES Y LOS CAMBIOS EN EL MODELO DE NEGOCIOS DEL SECTOR COMERCIAL DE ELECTRODOMÉSTICOS DE GUAYAQUIL
}

\section{ADOPTION OF STRATEGIES IN DIGITAL PLATFORMS AND CHANGES IN THE BUSINESS MODEL OF THE COMMERCIAL SECTOR OF ELECTRICAL APPLIANCES OF GUAYAQUIL}

\author{
Kelly Johanna Estrada Realpe ${ }^{1}$
}

Palabras clave:
Innovación,
Tecnología,
Plataformas
Digitales

\section{Resumen}

El presente trabajo tiene como objetivo analizar las estrategias y acciones adoptadas, en plataformas virtuales, por empresas comercializadoras de electrodomésticos en la ciudad de Guayaquil. Para este análisis se abordaron teorías relacionadas con el enfoque de las plataformas digitales en los negocios, los modelos de negocios existentes y el modelo de negocios CANVAS. Este último modelo se tomó como referencia para la elaboración de una guía de observación, la misma que fue aplicada a un total de 10 empresas dedicadas a la comercialización de electrodomésticos en la ciudad de Guayaquil. Esta guía de observación estuvo compuesta por un total de 49 ítems agrupadas en las 9 dimensiones del modelo CANVAS. Los resultados más relevantes determinan que la mayoría de estos negocios han optado, de manera reactiva, a utilizar a las redes sociales como medios publicitarios que motiven a la compra de los diferentes productos, ya sea en el establecimiento físico o en el canal e-commerce que varios negocios implementaron a partir de la pandemia por Covid-19.

Códigos JEL: E30, L21

\begin{abstract}
The objective of this work is to analyze the strategies and actions adopted, in virtual platforms, by commercial appliance companies in the city of Guayaquil. For this analysis, theories related to the approach of digital platforms in business, existing business models and the CANVAS business model were addressed. This last model was taken as a reference for the elaboration of an observation guide, which was applied to a total of 10 companies dedicated to the commercialization of electrical appliances in the city of Guayaquil. This observation guide was made up of a total of 49 items grouped in the 9 dimensions of the CANVAS model. The most relevant results determine that most of these businesses have reactively chosen to use social networks as advertising means that motivate the purchase of different products, either in the physical establishment or in the electronic commerce channel. that several companies implemented from the Covid-19 pandemic.
\end{abstract}

\footnotetext{
${ }^{1}$ Universidad Espíritu Santo - Ecuador.

E-mail: kjestrada@uees.edu.ec
} 


\section{INTRODUCCIÓN}

La llegada de la pandemia mundial del Covid-19 trajo diversos efectos para el año 2020, como resultado de las medidas de contingencia tomadas por los gobiernos de turno, las mismas que generaron uno de los cambios más radicales en las diversas áreas de la vida de una persona (Instituto de Investigaciones Económica (IIE), 2020). Estas medidas también ocasionaron problemáticas en los mercados y diferentes negocios, dado que ante la posibilidad de quedarse sin clientes y no generar ingresos, muchas organizaciones se vieron en la necesidad de replantear sus estrategias comerciales para mantenerse activas durante el tiempo de cuarenta para captar recursos económicos que le permitan solventar sus costos de operación (CEPAL, 2020).

Por esta razón, diversos negocios han diseñado importantes cambios estructurales para encarar las amenazas que derivan de la desaceleración de la demanda, como resultado de un estado de confinamiento, medidas de distanciamiento e inclusive restricciones en la movilidad vehicular y de personas (Pacto Mundial, 2020). Durante el tiempo de pandemia, el internet ha sido el principal aliado de los negocios y esto se evidencia en el auge que ha tenido en los últimos años en Ecuador, donde la tasa de acceso a internet pasó de $31 \%$ en 2012 , hasta $81 \%$ en el 2019 (Instituto Nacional de Estadísticas y Censos, 2019).

Peregrina (2017) argumenta que el crecimiento exponencial del internet ha significado una oportunidad muy atractiva para todo negocio que espera mantenerse en el mercado y proyectar un desarrollo económico. Las empresas que utilizan estas herramientas tecnológicas son capaces de tener un acercamiento más personalizado con sus clientes (Santos, 2020), a tal punto que dicha interacción les facilita la recogida de datos relevantes para el desarrollo de una estrategia digital que capte el interés del consumidor en línea, y así cumplir sus expectativas, al mismo tiempo que éste permite también alcanzar las metas empresariales.

El estado de excepción y las medidas de confinamiento generaron mucha preocupación en el sector empresarial ecuatoriano, el cual se dedica mayormente a la actividad comercial. Los datos del Instituto Nacional de Estadísticas y Censos (2018) establecen que, de un total de 884 mil establecimientos registrados en el país, el 36\% tiene como actividad económica el comercio al por mayor y menor de todo tipo de bienes, siendo tres las categorías destacadas: (a) alimentos y bebidas, (b) vehículos y (c) electrodomésticos (Instituto Nacional de Estadísticas y Censos, 2020). En este sentido, la investigación intenta analizar el aporte que el uso de plataformas digitales trajo consigo para un grupo de negocios dedicados a la venta de electrodomésticos en la ciudad de Guayaquil, tomando en consideración que se trata de la tercera categoría más importante dentro del sector comercial.

Sin embargo, la problemática de estudio se relaciona con la escasa literatura existente sobre los cambios que han generado el uso de plataformas digitales en el sector comercial de Guayaquil, y los desafíos que deben enfrentar algunos administradores al momento de apostarle a una estrategia digital (Cámara Ecuatoriana de Comercio Electrónico, 2021), aun cuando no poseen conocimientos sobre qué tipo de acciones deben implementar para captar clientes y mantenerse operativos dentro de un sector tan competitivo como es la venta de electrodomésticos.

Por esta razón, el objetivo del trabajo es analizar las estrategias y acciones adoptadas en plataformas digitales y los cambios que han generado en el modelo de negocios del sector comercial de electrodomésticos de la ciudad de Guayaquil. Para lo cual es importante hacer un estudio de carácter cualitativo que, mediante una guía de observación, recoja los cambios más relevantes sobre la actual estrategia comercial que se aplica en tiempos de pandemia, para hacer un contraste con las acciones aplicadas previo al Covid-19.

De acuerdo con la Cámara Ecuatoriana de Comercio Electrónico (2020) el Covid-19 forzó a que los consumidores realicen sus compras habituales, a través de canales virtuales, y esto implicó también que las empresas dispongan de plataformas digitales que sean capaces de atender 
los requerimientos de los clientes (Alvarado \& Vergara, 2018).

Finalmente, esta investigación es importante porque las plataformas digitales que utilizan internet, ya daban indicios de convertirse en los medios de comunicación del futuro, las cuales gracias a los avances tecnológicos, empezaron a captar el interés del sector empresarial; a tal punto que hoy por hoy, compañías como Amazon, dan muestra del éxito que puede tener un negocio que utiliza canales virtuales para realizar la compra y venta de todo tipo de bienes y servicios (Martínez et al., 2010).

\section{METODOLOGÍA}

Para el análisis de la situación actual que atraviesan las empresas del sector comercial de electrodomésticos de Guayaquil, esta investigación se desarrolló bajo un diseño no experimental, debido a que no se manipularon las variables que forman parte del estudio (Hernández et al., 2016). Asimismo, la investigación es de alcance longitudinal porque los datos se recogieron en varios períodos de tiempo, para comparar cómo han evolucionado estos negocios antes y durante la pandemia del Covid-19.

Para el levantamiento de datos, la población objeto de estudio fue delimitada por el total de compañías dedicadas a la actividad de comercialización de electrodomésticos, clasificadas dentro de la codificación CIIU G4649.11. De acuerdo con la Superintendencia de Compañías (2020) hasta el año 2019 se registraron 120 establecimientos dentro de esta codificación en la ciudad de Guayaquil. No obstante, para efectos de este estudio se escogió a un total de 10 compañías, que representan el $8 \%$ de la población.

La técnica de muestreo que se aplicó fue no probabilística - por conveniencia, dado que se extrajo de forma aleatoria a un grupo de 10 compañías del sector comercial de electrodomésticos, las mismas que cumplían las siguientes condiciones: (a) tenían al menos 10 años en el mercado, (b) tenían locales en Guayaquil o hacían entregas en esta ciudad y (c) contaban con presencia en plataformas digitales, ya sea mediante página web, redes sociales o ambas. Además, dentro de las 10 compañías seleccionadas se tuvo una persona que sirvió de contacto para la realización de las diferentes consultas que permitieron completar la guía de observación.

La toma de datos fue posible gracias al diseño de una guía de observación compuesta por un total de 49 ítems o preguntas que estuvieron distribuidas dentro de los nueve componentes del modelo de negocios CANVAS. De esta manera, el instrumento permitió recoger información relevante de cada uno de los 10 establecimientos objeto de estudio, para hacer un contraste de las estrategias de negocios adoptadas antes y durante los efectos de la pandemia del Covid-19.

La técnica de levantamiento de datos fue la observación, la misma que se enfocó en analizar la presencia que tienen estas 10 compañías en medios digitales; es decir, si disponían de una página web corporativa, si tenían la opción de realizar compras en línea, y si utilizaban redes sociales para interactuar con los clientes actuales y potenciales. Finalmente, la técnica empleada para el análisis de datos fue la sistematización de la información, debido a que los datos recopilados dentro de las guías de observación fueron estructurados en función de los nueve componentes del modelo CANVAS, a fin de hacer una interpretación general de los hallazgos más importantes de las compañías del sector comercial de electrodomésticos. A continuación, se presentan los ítems que formaron parte del instrumento aplicado.

\section{RESULTADOS}

Para poder llevar a cabo la investigación se tomó como referencia una guía de observación basada en el modelo de negocio CANVAS, la misma que fue aplicada a un total de 10 empresas del sector comercial de electrodomésticos de Guayaquil: Mega Créditos JR, FM Store, Importadora Jarrín, Almacenes Point, Almacenes Jaher, Créditos Económicos, La Ganga, Comandato, Artefacta y Japón. El objetivo de esta guía es poder hacer un contraste sobre la estrategia que han adoptado estos negocios en el contexto digital para hacer frente a los cambios que trajo consigo la pandemia del Covid-19. 


\section{CONCLUSIONES}

La pandemia del Covid-19 trajo consigo una transformación en el modelo de negocios de un sinnúmero de establecimientos, pero para efectos de este estudio se delimitó en la situación de aquellos dedicados a la comercialización de electrodomésticos. Normalmente, la mayoría se dedicaba a la venta de sus productos a través de canales tradicionales, y aunque tenían presencia en el entorno digital, este no era muy aprovechado. Una vez que las medidas de confinamiento y restricción de movilidad se pusieron en marcha en el Ecuador, los negocios tuvieron que replantear sus esquemas y procesos de operación, siendo el e-commerce una alternativa viable para garantizar la sostenibilidad de estos negocios en el corto y mediano plazo.

Para efectos de este análisis fue necesario construir una guía de observación basada en los nueve componentes del modelo CANVAS, lo que facilitó en la recopilación de datos, a su vez que permitió tener un mejor enfoque, a través de un estudio cualitativo, donde se trató de profundizar la mayor cantidad de detalles posibles, de cada uno de los diez negocios objeto de estudio.

Como conclusión general de esta investigación, se pudo determinar que prácticamente todos los 10 establecimientos que formaron parte del estudio han volcado su estrategia comercial en el contexto digital. La forma en que esto se evidencia es el uso de las redes sociales como un canal para desarrollar una interacción y comunicación más directa con sus clientes, para motivarlos a realizar una compra en el canal e-commerce de estos negocios. Del total de negocios consultados, se determinó que solo uno no disponía de una plataforma de compra en línea, pero todos ofrecen entrega a domicilio y tienen presencia en medios digitales, ya sea a través de su página web o las redes sociales más populares del medio como es el caso de Facebook e Instagram.

\section{Fortalezas y Limitaciones.}

La fortaleza de este estudio se relaciona con la posibilidad de contar con una guía de observación que hizo que se tengan mayores detalles de la forma en que estas 10 empresas del sector comercial de electrodoméstico han adoptado una estrategia digital para tener un mayor apego e interacción con sus clientes, para mantenerse activas en el mercado. Mientras que una limitación tuvo que ver con la muestra, dado que al tratarse de un estudio cualitativo es muy complejo analizar a un mayor número de compañías por el tiempo que tardó cada ficha en elaborarse, dedicándose más de 4 horas por cada compañía.

\section{Futuras Líneas de Investigación.}

Finalmente, como futuras líneas de investigación se recomienda la aplicación de la presente guía de observación basada en el modelo de negocios CANVAS, pero adaptándola a la actividad económica de otros sectores, tales como: servicios profesionales, restaurantes, farmacias, entre otros; a fin de comprender de una manera más detalla la estrategia que estos negocios adoptaron en tiempos de pandemia para reactivarse económicamente.

\section{REFERENCIAS}

Alvarado, Á., \& Vergara, N. (2018). El desafío del comercio electrónico en la economía del Ecuador. Polo Del Conocimiento, 3(23), 6786. https://doi.org/10.23857/pc.v3i1

Barullas, J. (2016). El comportamiento del consumidor y las nuevas tendencias de consumo antes las TIC. Economía y Empresa, 1(1), 10-18.

Cámara Ecuatoriana de Comercio Electrónico. (2020). Transacciones electrónicas en Ecuador durante el Covid-19. https://cece.ec/wpcontent/uploads/2020/06/Transaccioneselectronicas-en-Ecuador-durante-elCovid19.pdf

Cámara Ecuatoriana de Comercio Electrónico. (2021). Comportamiento de las transacciones no presenciales en Ecuador $2019-2020 . \quad \mathrm{https} / / / \mathrm{cece} . \mathrm{ec} / \mathrm{wp}-$ content/uploads/daeuploads/PresentacionMedicionEcommerce2 020-UEES-04MAYO2020.pdf

CEPAL. (2020). COVID-19 tendrá graves efectos 
sobre la economía mundial e impactará a los países de América Latina y el Caribe. https://www.cepal.org/es/comunicados/covi d-19-tendra-graves-efectos-la-economiamundial-impactara-paises-america-latina

Ferrer, L. (2018). Comportamiento del consumidor 2.0: nuevas realidades en entornos digitales. Marketing Visionario, 1(2), 141-155.

Rojas, M., Arango, P., \& Gallego, J. (2009). Trust in online purchasing. DYNA (Colombia), 76(160), 263-272.

Sánchez, J., \& Montoya, L. (2017). La confianza como elemento fundamental en las compras a través de canales de comercio electrónico : caso de los consumidores en Antioquia (Colombia). Revisa Innovar, 27(64), 11-22. https://doi.org/10.15446/innovar.v27n64.62 365.CITACI

Santos, I. (2020). El comportamiento del consumidor y las nuevas tendencias de consumo ante las TIC. Economic and Business Journal, 50(3), 599-620. https://doi.org/10.7200/esicm.164.0503.4 


\section{APÉNDICES}

Tabla 1.

Elementos de la guía de observación (parte 1)

\begin{tabular}{|c|c|c|}
\hline Segmentación de clientes & Propuesta de Valor & Canales de Distribución \\
\hline $\begin{array}{ll}\text { - } & \text { Edad promedio de los } \\
\text { clientes } \\
\text { - } & \text { Género de la mayoría de } \\
\text { clientes } \\
\text { - } \quad \text { Ubicación del } \\
\text { establecimiento } \\
\text { - Mercado Meta }\end{array}$ & $\begin{array}{ll}\text { - } & \text { Marcas que comercializa } \\
\text { - } & \text { Marcas más vendidas } \\
\text { - } & \text { Tipo de electrodomésticos que } \\
& \text { más se vende } \\
\text { - } & \text { Relación calidad - precio } \\
\text { - } & \text { Factor diferenciador del negocio }\end{array}$ & 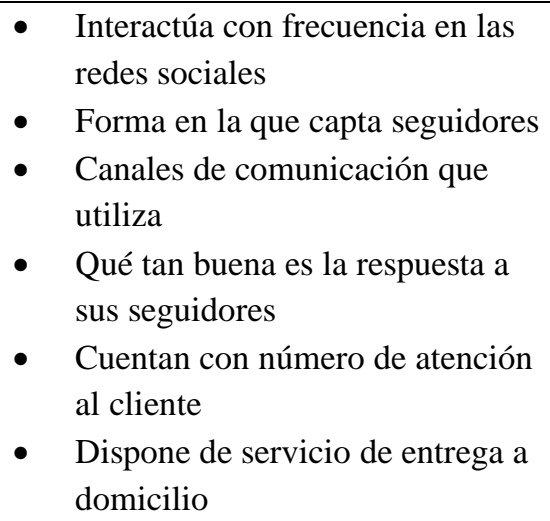 \\
\hline Relación con los clientes & Estructura de ingresos & Actividades Claves \\
\hline $\begin{array}{ll} & \text { Responde ágilmente a } \\
& \text { los clientes } \\
\text { - } & \text { Tiempo que toma en } \\
& \text { responder a algún } \\
& \text { requerimiento } \\
\text { - } & \text { Las respuestas en medio } \\
& \text { sociales son aplicables a } \\
\text { todo público } \\
\text { Quién responde los } \\
\text { mensajes }\end{array}$ & $\begin{array}{ll}\text { - } & \text { Porcentaje de ventas que } \\
\text { proviene de canales virtuales } \\
\text { - } & \text { El local es propio o alquilado } \\
\text { - } & \text { Existe algún ingreso indirecto } \\
\text { - } & \text { Existe alguna comisión que gane } \\
\text { - } & \text { El negocio } \\
\text { - } & \text { alguna marca } \\
\text { Cobran por el servicio de entrega } \\
\text { a domicilio }\end{array}$ & $\begin{array}{l}\text { - } \begin{array}{l}\text { Frecuencia de la creación de } \\
\text { - }\end{array} \\
\text { - } \text { Gradenido de atractivo del contenido } \\
\text { - } \text { promoción de público a través de } \\
\text { - } \text { Pagan publicidad en otros medios } \\
\text { - } \quad \text { Lalidad de la atención al cliente } \\
\text { - Siguen tendencias en el mercado } \\
\text { - Sorteos y give aways son } \\
\quad \text { actividades importantes }\end{array}$ \\
\hline Recursos Claves & Alianzas Estratégicas & Estructura de Costos \\
\hline $\begin{array}{l}\text { - Número de personas que } \\
\text { labora en el local } \\
\text { - Tipo de infraestructura } \\
\text { física que posee el local } \\
\text { - Tiene vehículos para } \\
\text { entregar el producto al } \\
\text { cliente }\end{array}$ & $\begin{array}{ll}\text { - } & \text { Tiene alianza con influencers } \\
\text { - } & \text { Tiene alianzas con distribuidoras } \\
\text { - } & \text { Tiene algún auspiciante o } \\
\text { - } & \text { patrocinio de su marca } \\
& \text { empresas }\end{array}$ & $\begin{array}{l}\text { - Tiene bien definida su estructura } \\
\text { de costos } \\
\text { - Tiene identificado sus costos } \\
\text { fijos } \\
\text { - Los precios que maneja permiten } \\
\text { generar una rentabilidad óptima } \\
\text { - Hay variación de costos mes a } \\
\text { mes } \\
\text { Hay costos de oportunidad que } \\
\text { no se han considerado }\end{array}$ \\
\hline
\end{tabular}


Tabla 2.

Segmentación de clientes (Parte 1)

\section{COMPONENTES}

Segmentación de clientes

\section{RESULTADOS OBSERVADOS}

El target de Mega Créditos está conformado por personas jóvenes, entre los 25 y 45 años de edad. Aunque no hay restricción de género, se nota una mayor preferencia de la marca por parte de los hombres. Un aspecto esencial es que están ubicados tanto en la ciudad de Guayaquil, como en tres cantones de la provincia de El Oro como: Machala, El Guabo y Pasaje. Sus productos se enfocan a personas de nivel socioeconómico medio.

El target de FM Store se compone de gente joven, entre los 18 y 35 años de edad. En sus plataformas se notó una interacción frecuente de mujeres, aunque no tiene una restricción de género, los productos son aptos para todo público. Su mercado principal es Guayaquil, pero sí disponen de entrega a nivel nacional, dado que sus productos tienen un enfoque socioeconómico medio.

Importadora Jarrín tiene un target más maduro porque en este almacén compran personas de edades entre los 30 y 40 años. Está enfocado a un nivel socioeconómico medio - bajo de la ciudad de Guayaquil, teniendo un local en el centro de Guayaquil

El target de Almacenes Point está dirigido a personas entre 20 - 45 años. A pesar de no tener restricción de género, se notó una mayor interacción del público masculino. Cuenta con 44 locales a nivel nacional y se enfoca mayormente a personas de NSE medio.

El target de Almacenes Jaher está compuesto por personas de 25 a 45 años de edad, mayormente de la ciudad de Guayaquil, de nivel socioeconómico medio. Cuenta con establecimientos en el norte, centro y sur de la urbe. No tiene restricción de género, pero interactúan más hombres que mujeres

El target de Créditos Económicos se compone mayormente por personas entre los 30 y 50 años, es decir, un mercado adulto y maduro, dado que se trata de una cadena de almacenes tradicional en el país. No tiene restricción de género en cuanto a su enfoque, y tiene presencia importante en los centros comerciales más reconocidos de Guayaquil. Por esta razón, su segmento es dirigido a personas de un nivel socioeconómico medio alto. 
Tabla 3.

Segmentación de clientes (Parte 2)

\begin{tabular}{|c|l|}
\hline \multirow{5}{*}{$\begin{array}{c}\text { Segmentación } \\
\text { de clientes }\end{array}$} & $\begin{array}{l}\text { El target de Almacenes La Ganga está compuesto mayormente por personas entre los } 30 \text { y } 50 \\
\text { años, es decir, un mercado adulto y maduro, dado que se trata de una cadena de almacenes } \\
\text { tradicional en el país. No tiene restricción de género en cuanto a su enfoque, y tiene presencia } \\
\text { importante en los centros comerciales más reconocidos de Guayaquil. Por esta razón, su } \\
\text { segmento es dirigido a personas de un nivel socioeconómico medio - alto. } \\
\text { El Target de Comandato se compone mayormente por personas entre los } 30 \text { y } 50 \text { años, es } \\
\text { en el país. No tiene restricción de género en cuanto a su enfoque, y tiene presencia importante } \\
\text { en los centros comerciales más reconocidos de Guayaquil. Por esta razón, su segmento es } \\
\text { dirigido a personas de un nivel socioeconómico medio - alto. } \\
\text { Artefacta no tiene realmente una distinción en cuanto a edad, pero se observó que en sus } \\
\text { canales virtuales interactúan tanto hombres y mujeres de edades entre 30 y 50 años, es decir, } \\
\text { un público joven - adulto. Tiene una importante presencia en centros comerciales de la } \\
\text { ciudad de Guayaquil, así como locales en zonas estrategias tanto en el norte, sur y centro, y el } \\
\text { precio de sus productos está direccionado a un target de nivel socioeconómico medio y alto. } \\
\text { El target de Almacenes Japón se caracteriza por hombres y mujeres de la ciudad de } \\
\text { Guayaquil, de estratos sociales medio y altos, cuyas edades oscilan entre los } 25 \text { y 50 años; es } \\
\text { decir, un público joven - adulto. }\end{array}$ \\
\hline
\end{tabular}

Tabla 4.

Propuesta de Valor

\begin{tabular}{|l|l}
\hline Propuesta de Valor & $\begin{array}{l}\text { En cuanto a la propuesta de valor de estos almacenes es muy similar, dado que todos } \\
\text { comercializan electrodomésticos de buena calidad, aunque su catálogo de productos está } \\
\text { conformado mayormente por marcas como Indurama, Sony, Whirlpool, Huawei, Nokia, } \\
\text { Lenovo,TCL, Riviera, Dell, HP, Samsung, LG, Global y Tundra, especializada en venta de } \\
\text { motos también. Aunque comercializa productos de línea blanca como refrigeradoras, } \\
\text { cocinas, lavadoras y acondicionadores de aire. Pero su factor diferenciador es la } \\
\text { posibilidad de otorgar crédito directo a las personas, según su estatus crediticio. Mega } \\
\text { Créditos no posee un canal e-commerce, pero sí utiliza las redes sociales para interactuar } \\
\text { con sus clientes y concretar ventas, así como coordinar pedidos y facturación; los otros } \\
\text { nueve establecimientos sí dispone de un canal de ventas online. }\end{array}$
\end{tabular}

Tabla 5.

Canales de Distribución

\begin{tabular}{l|l} 
Canales de & Todos los negocios consultados tienen una importante interacción en medios digitales
\end{tabular} distribución $\quad$ como las redes sociales, donde interactúan con sus clientes teniendo una respuesta mayormente ágil, pues se responde a los mensajes en cuestión de 15 minutos como máximo. Si bien todos los negocios disponen de un local físico, también disponen de un canal e-commerce, a excepción de Mega Créditos, que podría decirse es el menos competitivo de los nueve restantes. Lo que les da la oportunidad de ofrecer un servicio de compra $24 / 7$ y una entrega coordinada a nivel nacional, sea por flota propia o subcontratando una compañía de transporte como Servientrega, lo que da la oportunidad de entregar los pedidos en un lapso entre 1 y 3 días como máximo dependiendo de la zona de entrega, el valor del recargo por transporte puede ser gratuito o costar hasta $\$ 70.00$ como máximo. 
Tabla 6.

Relación con los clientes

Relación con los $\quad$ En establecimientos más nuevos como Point y FM Store, la interacción es plataformas

clientes $\quad$ digitales es alta, especialmente en Facebook, dado que tienen un Community Manager que se encarga de la gestión de redes. La respuesta es ágil, pues demoran menos de 15 minutos en responder. En cuanto a las cadenas más tradicionales como Japón, Comandato, Artefacta y Créditos Económicos la interacción en redes sociales no es tan alta, dado que esta se genera mayormente en sus locales físicos.

Tabla 7.

Estructura de Ingresos

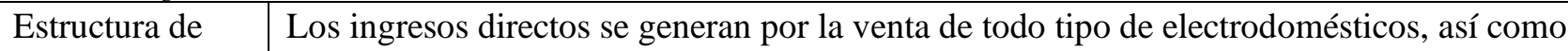

Ingresos $\quad$ también de celulares, computadoras y equipos de audio y video, a través de su canal ecommerce y/o locales físicos ubicados en centros comerciales o zonas estratégicas de la ciudad de Guayaquil.

Tabla 8.

Actividades Claves

\begin{tabular}{|l|l|}
\hline Actividades claves & $\begin{array}{l}\text { Todos estos establecimientos realizan al menos } 3 \text { publicaciones semanales en Facebook, } \\
\text { las cuales tienen en promedio más de } 200 \text { likes y más de } 50 \text { interacciones. Estas } \\
\text { plataformas se vuelven en espacios que captan el interés del consumidor para realizar } \\
\text { compras en el canal virtual del negocio. Es decir, las redes sociales se vuelven en una } \\
\text { plataforma para incentivar la compra en canales virtuales, así como para obtener } \\
\text { información relevante de los clientes y diseñar estrategias personalizadas que sean capaces } \\
\text { de satisfacer sus necesidades. } \\
\text { También, realizan promociones de acuerdo con la temporada, por ejemplo, san Valentín, } \\
\text { día de la madre, día del padre y navidad. Pagan publicidad en medios televisivos y } \\
\text { radiales, así como Vallas publicitarias. Realizan sorteos en épocas de temporadas altas, } \\
\text { como día de la madre o navidad. }\end{array}$ \\
\hline
\end{tabular}

Tabla 9.

Recursos Claves

Recursos claves

Tiene locales físicos en varios puntos de Guayaquil para dar a conocer sus productos. La entrega se hace a través de una compañía de transporte contratista o por medio de una flota de camiones propia. Así como también tienen personal suficiente para la logística de distribución de electrodomésticos a nivel nacional. 
Tabla 10.

Alianzas Estratégicas

\begin{tabular}{|c|l|}
\hline $\begin{array}{c}\text { Alianzas } \\
\text { estratégicas }\end{array}$ & $\begin{array}{l}\text { Algunas compañías como Almacenes Point, tienen alianza con Servientrega, lo que es un } \\
\text { factor diferenciador también pues, esto garantiza una entrega en todo el país. Otros } \\
\text { almacenes tienen alianzas con distribuidores y los fabricantes directos, lo que hace posible } \\
\text { que dispongan de precios más competitivos. } \\
\text { En el caso de Almacenes La Ganga, el influencer más destacado es David Reinoso con su } \\
\text { personaje "El Cholito" y Flor María Palomeque. Es uno de los auspiciantes de Barcelona } \\
\text { Sporting Club. Se observa que en Artefacta la imagen del cantante ecuatoriano Aladino } \\
\text { (Norberto Vargas) es utilizada como influencer, dado que lo disfrazan de como genio en } \\
\text { temas de descuentos y demás ofertas que realiza el almacén. Además, tienen alianza con } \\
\text { distribuidores e importadores, por eso tienen precios competitivos. Los demás negocios no } \\
\text { tienen un influencer identificable. }\end{array}$ \\
\hline
\end{tabular}

Tabla 11.

Estructura de Costos

Estructura de

Los costos se aplican en función de la compra de los electrodomésticos que se costos comercializan y le agregan un margen de ganancia, para competir según los precios del mercado. Los costos pueden variar según las políticas que se apliquen a los bienes de consumo, como salvaguardias, aranceles, y demás costos de importación y transporte que pudieran darse dentro de la cadena de abastecimiento. 